\title{
Using a deliberative forum for engaging health system and health plan leaders to prioritize research topics
}

\author{
Kathleen M. McTigue MD MPH ${ }^{a}, E$. Johanna Hartelius PhD ${ }^{b}$, Timothy S. Anderson MD ${ }^{c}$
} Andrew P. Allsup ${ }^{d}$, Treva Alston MS ${ }^{e}$, Cynthia H. Chuang MD MSc ${ }^{f}$, Stacey Dillon MS MA ${ }^{g}$, Daniel E. Ford MD MPH ${ }^{h}$, Nivedita Gunturi MDi, Rachel Hess MD MS ${ }^{j}, H$. Lester Kirchner $\mathrm{PhD}^{\mathrm{k}}$, Sharon L. Larson PhD', Anita B. Leon-Jhong $\mathrm{MD}^{\mathrm{m}}$, David R. McCoy MA ${ }^{\mathrm{n}}$, Anuradha Paranjape MD MPH${ }^{\circ}$, James R. Uhrig ${ }^{p}$, Anam A. Waheed $\mathrm{MD}^{q}$ and Gordon R. Mitchell PHD

a Associate Professor of Medicine \& Epidemiology \& Director, Clinical Scientist Track for Internal Medicine Residents, Department of Medicine, University of Pittsburgh School of Medicine, Pittsburgh, PA, USA

b Associate Professor, Department of Communication Studies, University of Texas at Austin, Austin, TX, USA

c Primary Care Research Fellow, Division of General Internal Medicine, University of California San Francisco, San Francisco, CA, USA

d Graduate Student, Department of Communication, University of Pittsburgh, Pittsburgh, PA, USA

e Patient Partner, PaTH Network, Johns Hopkins University, Baltimore, MD, USA

f Professor of Medicine \& Public Health Services, Division of General Internal Medicine, Penn State College of Medicine, Hershey, PA, USA

g Behavioral Health Outpatient Therapist, Pittsburgh, PA, USA

$\mathrm{h}$ Professor of Medicine, Department of Medicine, Johns Hopkins University, Baltimore, MD, USA

i Geriatric Medicine Fellow, University of Pittsburgh Medical Center (UPMC), Pittsburgh, PA, USA

j Chief, Division of Health System Innovation and Research, University of Utah, Salt Lake City, UT, USA

k Professor, Department of Biomedical and Translational Informatics, Geisinger Health System, Danville, PA, USA

I Professor, College of Population Health, Thomas Jefferson University, Philadephia, PA, USA

$\mathrm{m}$ Clinical Instructor of Medicine Division of General Internal Medicine, University of Pittsburgh Medical Center (UPMC), Pittsburgh, PA, USA

n Graduate Student, Department of Political Science, University of Pittsburgh, Pittsburgh, PA, USA

o Physician and Interim Vice Chair, Department of Medicine, Lewis Katz School of Medicine at Temple University, Philadelphia, PA, USA

p Patient Partner, PaTH Network, University of Pittsburgh, Pittsburgh, PA, USA

q Internal Medicine Resident, University of Pittsburgh Medical Center (UPMC), Pittsburgh, PA, USA

r Associate Professor, Department of Communication, University of Pittsburgh, Pittsburgh, PA, USA

\begin{abstract}
Background: Including stakeholders in the process and outcomes of comparative effectiveness research (CER) can help ensure that research questions are relevant and findings are communicated to individuals who need them for decisionmaking. Yet limited strategies are available to assist researchers with stakeholder engagement. While health system leaders' perspectives are increasingly recognized as valuable for CER planning, their inclusion in the stakeholder pool raises challenges due to differences in culture, training, incentives, priorities and language norms.

Objective: To convene and evaluate a deliberative forum for engaging health system leaders and other stakeholders in order to shape health system research priorities for the PaTH Clinical Data Research Network, a member of the National PatientCentered Clinical Research Network (PCORnet).

Methods: Breakout sessions and large group deliberation solicited diverse perspectives and explored benefits and challenges of different research questions. Topic reframing, narrative integration and dynamic updating techniques facilitated communication across diverse backgrounds. Participants included 29 health system and health plan leaders, clinicians, clinical researchers and patients from the network's 6 participating health systems. Main measures were audience response system (ARS) polling on general topic preferences and survey data on measures of engagement and deliberation success.

Results: A slate of 10 specific research topics was vetted; after deliberation, the group converged to favor the characterization of high utilizers of healthcare. Audience response polling revealed opinion shifts. Participants reported high levels of satisfaction with the experience and rated it highly for markers of deliberative quality (e.g., opportunity for active participation and adequate discussion, respect for others' opinions and awareness of different perspectives). Fifty-four percent noted their views on the issues changed. Most participants learned from the experience (93\%) and agreed that the process helped them to empathize with the challenges of others (85\%).

Conclusions: A deliberation forum can incorporate diverse stakeholders into CER, enabling participants to inform and learn from each other's perspectives while shaping a person-centered research trajectory.
\end{abstract}




\section{Keywords}

Comparative effectiveness research, communication, health priorities, health services research, patient-centered care, patient engagement, person-centered healthcare, stakeholder engagement

\section{Correspondence address}

Dr. Kathleen M. McTigue, 230 McKee Place, Suite 600, Pittsburgh PA 15213, USA. E-mail: kmm34@pitt.edu

Accepted for publication: 23 September 2017

\section{Introduction}

Stakeholder engagement in comparative effectiveness research (CER) aims to ensure that research questions are relevant and that stakeholders are involved throughout the research process [1,2]. Engagement may enhance study recruitment, promote greater uptake of research findings and facilitate decision-making in participatory design [1,3]. While stakeholder engagement is perceived as beneficial in fields such as community-based participatory research [4,5], environmental science [6] and energy policy [7-9], methods for incorporating it into CER were developed only recently [2]. Strategies for engaging stakeholders in the research design process are limited [10,11] and researchers have called for more descriptive studies on stakeholder engagement in health research [12].

Engaging a broad range of stakeholders is endorsed for CER [13,14] and scholars argue that patient-centered research should engage the spectrum of stakeholders involved in patient-centered healthcare (e.g., patients, clinicians, representatives from the delivery system and payers or purchasers) [13,15-17]. Yet, literature on CER and patient-centered research has focused primarily on patient engagement and little is known about engaging payers and key decision-makers at the health system level $[1,12]$. This project begins to fill that gap by bringing diverse stakeholders, including health system leaders, to the table. However, doing so poses challenges due to cultural factors, training, incentives, priorities, scheduling constraints, power differentials and language norms $[12,13]$.

The process of priority setting may particularly benefit from diverse stakeholder input, insofar as it promotes improved decision-making, stakeholder satisfaction and positive externalities such as media coverage or policy change [10,18]. Deliberative approaches to stakeholder engagement show particular promise for priority setting. Such events entail careful design of the deliberation process, an emphasis on facilitation and attention to the ways that stakeholders relate to each other $[19,20]$. Facilitators foster safe environments for information sharing, especially when stakeholder interests may be perceived as incompatible or when consensus must be achieved [11,21]. Distinct from survey or focus group approaches that elicit more initial and intuitive responses from participants, deliberative events aim to provide participants with information that is intentionally neutral and respectful of the full range of underlying values, experiences and possible perspectives. Participants are encouraged to discuss, learn from others and examine and refine their own views [22]. Collective problem solving is emphasized, along with exploration of different perspectives, consensus building and active participation by all stakeholders in a process that seeks to minimize status differentials $[11,20,23]$.

A limited literature suggests that facilitated public deliberation can successfully engage stakeholders to prioritize CER questions [10]. Various deliberative strategies have improved participant knowledge and influenced participant opinions [22]. In-person (versus remote) methods may provide more valued experiences [22].

We report here on the PaTH Health System Leader's Demonstration Project (HSLDP), which aimed to engage health system and health plan leaders, along with other stakeholders, in prioritizing CER questions. We describe and evaluate the deliberative process used to promote inperson dialogue, allow participants to listen to each other's perspectives in real time, reflect on and react to these perspectives and interact directly to reach a better understanding of the issues at hand.

\section{Methods}

\section{Overview}

The PaTH Clinical Data Research Network is a member of the National Patient-Centered Clinical Research Network (PCORnet) [24]. PaTH is a partnership between 6 health systems (Geisinger Health System, Johns Hopkins Health System, Penn State Milton S. Hershey Medical Center, Temple University Health System, UPMC and the University of Utah Health Care), their affiliated academic institutions and, in some cases, health plans. The HSLDP sought to engage health system leaders, clinicians and patients from across PaTH to gain insight about critical topics in health systems research and disseminate information about PCORnet. Goals included informing the development of a grant proposal seeking funds to answer the highest priority CER question and fostering longerterm collaboration with health system leaders.

A deliberative format was selected to foster learning among stakeholders. Health system and health plan leaders were invited to provide "content expertise," bringing direct expertise and insights on health system concerns. Patients, community engagement experts and clinicians, were invited as "citizen advisors" bringing values, patient perspectives and clinical context to the discussion. We 
engaged a facilitator team with expertise in public discourse and deliberative democracy.

Two facilitators, one clinician researcher and 2 patient partners planned the deliberative process. Special emphasis was placed on communicative practices to enact shared understanding across different backgrounds. Key challenges included ensuring that patient partners were empowered to participate, thereby reducing perceived power imbalances that can exist between them and experts [20] and fostering effective conversation between individuals with diverse backgrounds. The plan incorporated key features for priority setting in health services research, such as an explicit and transparent process, a focus on information management, a consideration of the context and values of all key stakeholder groups and a formal mechanism for reviewing decisions [18].

\section{Pre-event activities}

"Specific research topics" of importance to health systems were identified from the literature and by surveying PaTHaffiliated clinicians and health system leaders. We mapped these specific topics to more general strategies for improving health systems, expressed in language that is accessible to a non-technical audience (Table 1). Prior to the event, all patient stakeholders were invited to participate in a 2-hour telephone orientation to the deliberative goals and process. In addition, preparatory materials were distributed to participants, including an outline of the deliberation plan and topic-specific one-page briefs that summarized pertinent literature.

\section{Deliberation Event}

The deliberative event included 8 stages (Figure 1). Techniques were employed to heighten potential for emergence of "social knowledge" and group opinion formation. For example, interim opinion polling (after Stage 5) and participant-driven format revision for comparative deliberation (Stage 5) facilitated 'dynamic updating,' which can promote social learning and group opinion formation in deliberative exercises [25,26].

A 10-topic slate was the starting point for deliberation (Stage 1). Participants were tasked with narrowing the list to 2 to 3 top-priority topics. Patients/community advocates and clinicians were asked to focus attention on the general strategies for improving health systems. Conversely, health system and health plan leaders were asked to focus more on the specific research topics, which were framed in technical language to enable precise discussion. To moderate multiple forms of expertise, facilitators explicitly acknowledged that patients and clinicians were invited, but not expected, to participate in discussion of specific research topics.

\section{Figure 1 Summary of the deliberative process}

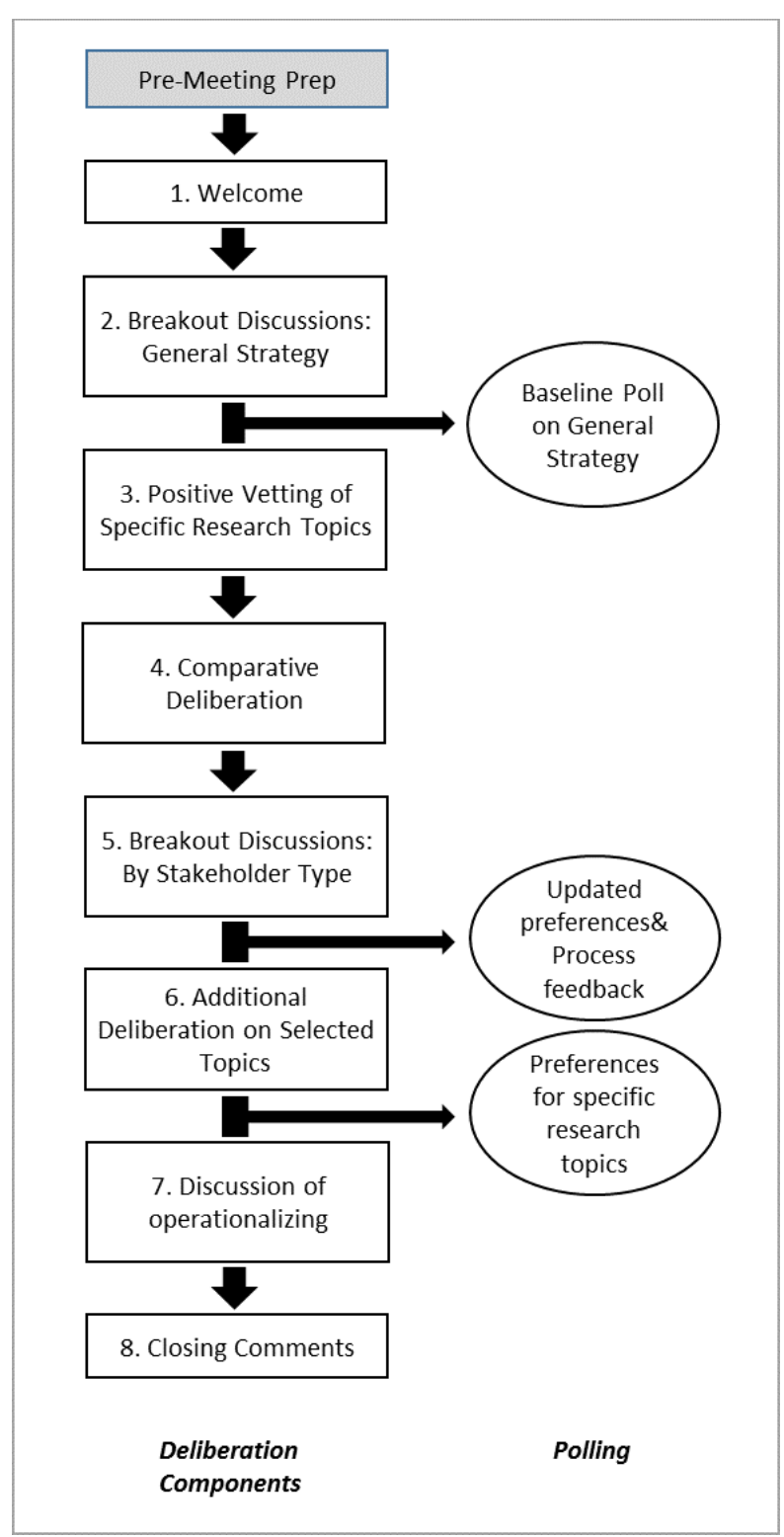

Deliberation began within break-out groups which included all 3 types of participants and concentrated on general research strategies (Stage 2). Patients were asked to open the discussion in order to frankly address the risk of perceived status inequities that might emerge. They set the tone for dialogue by identifying which general strategy was their top priority, illustrating their choice with a personal narrative. Such narrative communication facilitates mutual understanding across diverse frames of reference [27]. Open discussion among participants followed, focusing on how to prioritize among the general strategies. Each group's facilitator summarized the discussion in a report back to the larger group and then patient/community advocates and clinicians used ARS polling to identify their top-priority general strategy.

During positive vetting (Stage 3), for each specific research topic, participants were asked to identify potential benefits it could provide to health systems, clinicians, patients and patient families; to delineate how these 
Table 1 Summary of general strategies and specific research questions addressed in the deliberation

\begin{tabular}{|c|c|}
\hline General Strategies & Specific Research Questions \\
\hline \multirow[t]{5}{*}{$\begin{array}{l}\text { Helping healthcare providers make better } \\
\text { treatment recommendations }\end{array}$} & $\begin{array}{l}\text { A. Electronic Health Record (EHR) data should be used to monitor health system performance on } \\
\text { diverse measures of unnecessary care, as outlined in the "Choosing Wisely" initiative (e.g., } \\
\text { unnecessary antibiotic prescribing, unnecessary laboratory testing, unnecessary use of medical } \\
\text { imaging) }\end{array}$ \\
\hline & $\begin{array}{l}\text { B. EHR data should be used to characterize the diagnoses and procedures associated with } 23 \text { hour } \\
\text { admissions and determine whether subsequent healthcare utilization differs for similar patients } \\
\text { treated with and without a } 23 \text { hour admission approach }\end{array}$ \\
\hline & $\begin{array}{l}\text { C. Dispensing data held within EHRs should be used to quantify how often brand-name versus } \\
\text { generic drugs are used for patients treated in different healthcare settings (e.g., inpatient, } \\
\text { outpatient, emergency department). Further characterization by specific patient characteristics } \\
\text { will provide useful information to policy-makers aiming to promote the use of generic } \\
\text { medication }\end{array}$ \\
\hline & $\begin{array}{l}\text { D. Health record data should be utilized to identify patient/treatment characteristics which result in } \\
\text { admission } v s \text { discharge from the emergency department for common conditions (e.g., atrial } \\
\text { fibrillation) and whether implementation of clinical care pathways impact this decision }\end{array}$ \\
\hline & $\begin{array}{l}\text { E. EHR data should be utilized to determine the care gap that exists in the management of } \\
\text { osteoporosis and other diseases of aging. EHR can be used to provide automated notifications } \\
\text { to both PCPs and their patients of an existing care gap that could save millions in healthcare } \\
\text { costs via interventional or preventive care }\end{array}$ \\
\hline Making healthcare performance data more useful & $\begin{array}{l}\text { F. Existing health system metrics should be evaluated to determine which metrics predict } \\
\text { outcomes that matter to health system leaders (e.g., health outcomes, health system utilization) }\end{array}$ \\
\hline $\begin{array}{l}\text { Making sure that when people are discharged } \\
\text { from the hospital, they are ready and have the } \\
\text { help they need to continue to improve }\end{array}$ & $\begin{array}{l}\text { G. Health record data should be used to identify patient characteristics and clinical situations that } \\
\text { are associated with hospital re-admissions, enabling the development of policies that may } \\
\text { reduce re-admission risk }\end{array}$ \\
\hline \multirow[t]{3}{*}{$\begin{array}{l}\text { Understanding why some people use so much } \\
\text { more healthcare resources than others }\end{array}$} & $\begin{array}{l}\text { H. Longitudinal health record data should be used to characterize high-utilizers of healthcare } \\
\text { (general care or ED care), to identify which services they most often utilize and to examine } \\
\text { how their health service utilization evolves over time }\end{array}$ \\
\hline & $\begin{array}{l}\text { I. EHR data should be used to characterize patients with multiple co-morbidities and among } \\
\text { those patients, differentiate those with poor health outcomes from those with better health } \\
\text { outcomes }\end{array}$ \\
\hline & $\begin{array}{l}\text { J. EHR data should be used to characterize common end-of-life care and determine which } \\
\text { treatments are associated with both guideline-concordant care and reduced health service } \\
\text { utilization }\end{array}$ \\
\hline
\end{tabular}

benefits aligned with organizational priorities and to recognize when topics could be addressed with easily accessible data. This exercise aimed to generate an inventory of talking points for later PaTH advocacy of possible research topics to research funders. Stage 4, comparative deliberation, addressed reasons why some topics were superior to others, enabling participants to test the preliminary talking points. Facilitator cues steered participants to use constructive criticism to highlight limitations of the proposed research topics.

Additional break-out sessions allowed clinicians and patients/community advocates, in separate groups, to reflect on the prior discussion and prepare to provide the larger group with feedback on how well their perspectives had been represented (Stage 5). Concurrently, health system and health plan leaders used ARS polls to address how important further deliberation was for each specific research topic. This activity laid the groundwork for a deeper comparative discussion focused on vetting a select few of the research topics. Re-convening, clinicians and patients/community advocates were polled to see if perspectives had shifted regarding preferences for general research strategies. Participants in each stakeholder group also reported on how well they felt that their group's perspective was represented in the deliberation process. These polls helped the facilitators assess the need to adjust deliberation procedures.

A final round of whole-group deliberation (Stage 6) addressed the topics identified as needing further discussion by the health system/health plan leaders. 
Participants then voted on the specific research topic of highest priority. The event ended with a preliminary discussion of how to operationalize the prioritized topic and a summary of the planned decision-making process that would be used moving forward (Stage 7). That is, the final choice of specific research questions would be determined by PaTH's Steering Committee, after reflecting on data gathered throughout the deliberation event. To ensure communicative accountability, the final decision and rationale would be circulate to the entire group that had participated in the deliberation process. We assessed for opinion shift on general topics between the initial and follow-up audience-response using Chi-square tests. Because of the limited sample size, responses from patients, community advocates and clinicians were combined for this analysis. An exit poll provided a formal process evaluation [28], assessing markers of successful deliberation [22] and stakeholder engagement [20]. Data were summarized with descriptive statistics.

\section{Results}

Eleven health system leaders, 5 health plan leaders, 8 clinicians and 5 patients or community advocates gathered in Pittsburgh PA, on November 9, 2015, for the 4.5-hour deliberation event. Health system and health plan leaders' roles were diverse, such as hospital president, chief executive officer, chief information officer, director of behavioral health, vice president of operations and senior vice president. Baseline polls on general strategies showed that among the patients and community advocates, the preferred general strategy was helping healthcare providers make better treatment recommendations (80\%), with a minority selecting the topic focusing on adequate posthospitalization care (20\%; Figure 2). Among clinicians, $63 \%$ initially preferred a focus on understanding why some patients use more healthcare resources than others; 25\% favored a focus on ensuring that patients discharged from the hospital have the help they need to continue to improve and $13 \%$ preferred focusing on making healthcare performance data more useful.

The positive vetting process identified valuable aspects of all proposed topics. Comparative deliberation then focused on differentiating the relative value of topics - in particular, topics related to over-use of care and hospital re-admissions. In the second break-out session (Stage 5), health system/health plan leaders identified 3 topics for which further deliberation was important: (a) high utilizers of care (86\%); (b) health system metrics (69\%) and unnecessary care (56\%). Focused deliberation on this set of topics set the stage for final group polling.

Eleven participants (4 patients or community advocates and 7 clinicians) participated in the initial and follow-up polls assessing general strategy preference (1 patient and 1 clinician voted only at baseline). While $45 \%$ initially preferred the topic "Understanding why some people use so much more healthcare resources than others, 64\% preferred the same topic at the conclusion of deliberation $(p=0.015$; Figure 3$)$. The shift in responses occurred entirely among clinicians, with the 4 patients/community advocates maintaining consistent preferences.

\section{Figure 2 Responses to baseline poll on preferred general strategy. Participants answered the question "Which of the following seems most promising?"}

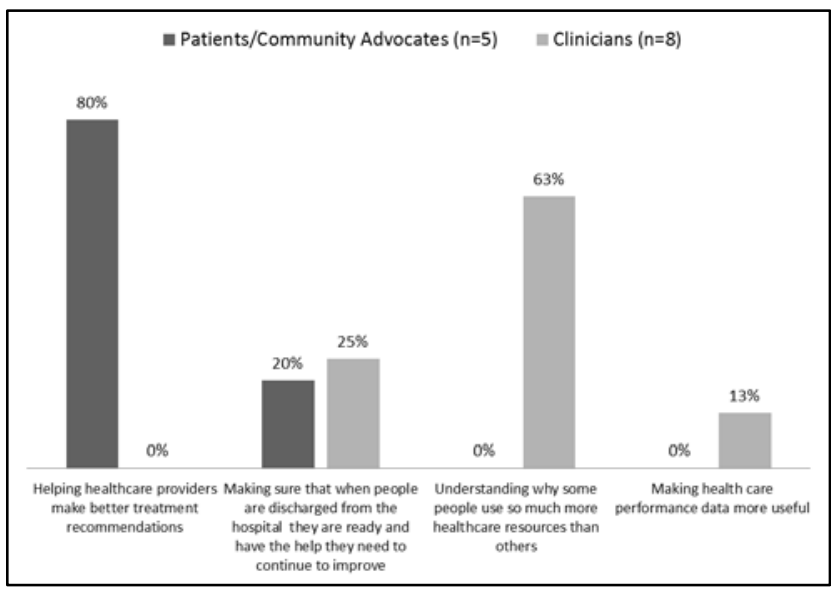

\section{Figure 3 Comparison of preferred strategy preferences prior to and following deliberation. Responses reflect 4 patients/community advocates and 7 clinicians}

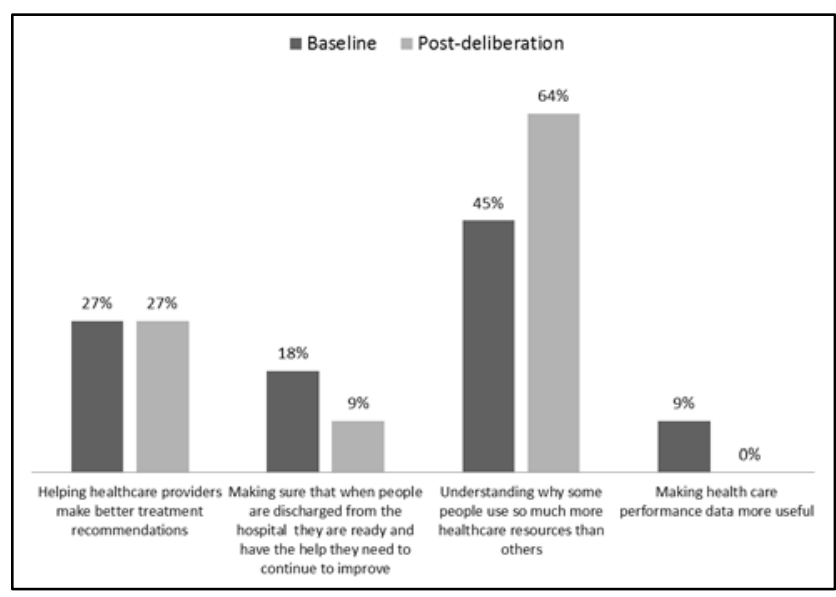

Despite a range of initial preferences for specific research topics, the final poll showed preferences converging on a single topic: "Understanding what drives high utilization of care" (55\% of respondents). Several other topics received a low percentage of votes (hospital re-admissions 14\%; health system metrics $14 \%$; unnecessary care $10 \%$; multiple comorbidities $3 \%$; care gaps for diseases of aging 3\%). Physical and mental health contributions to high use of care were both of high importance.

The interim process evaluation showed high satisfaction levels. When asked how they felt about the way that the patient perspective had been represented so far in the deliberation, $80 \%$ of patient respondents were 
Table 2 Perceptions about the deliberation event among the 26 participants who participated in the evaluation survey [frequency $(\%)$ ]

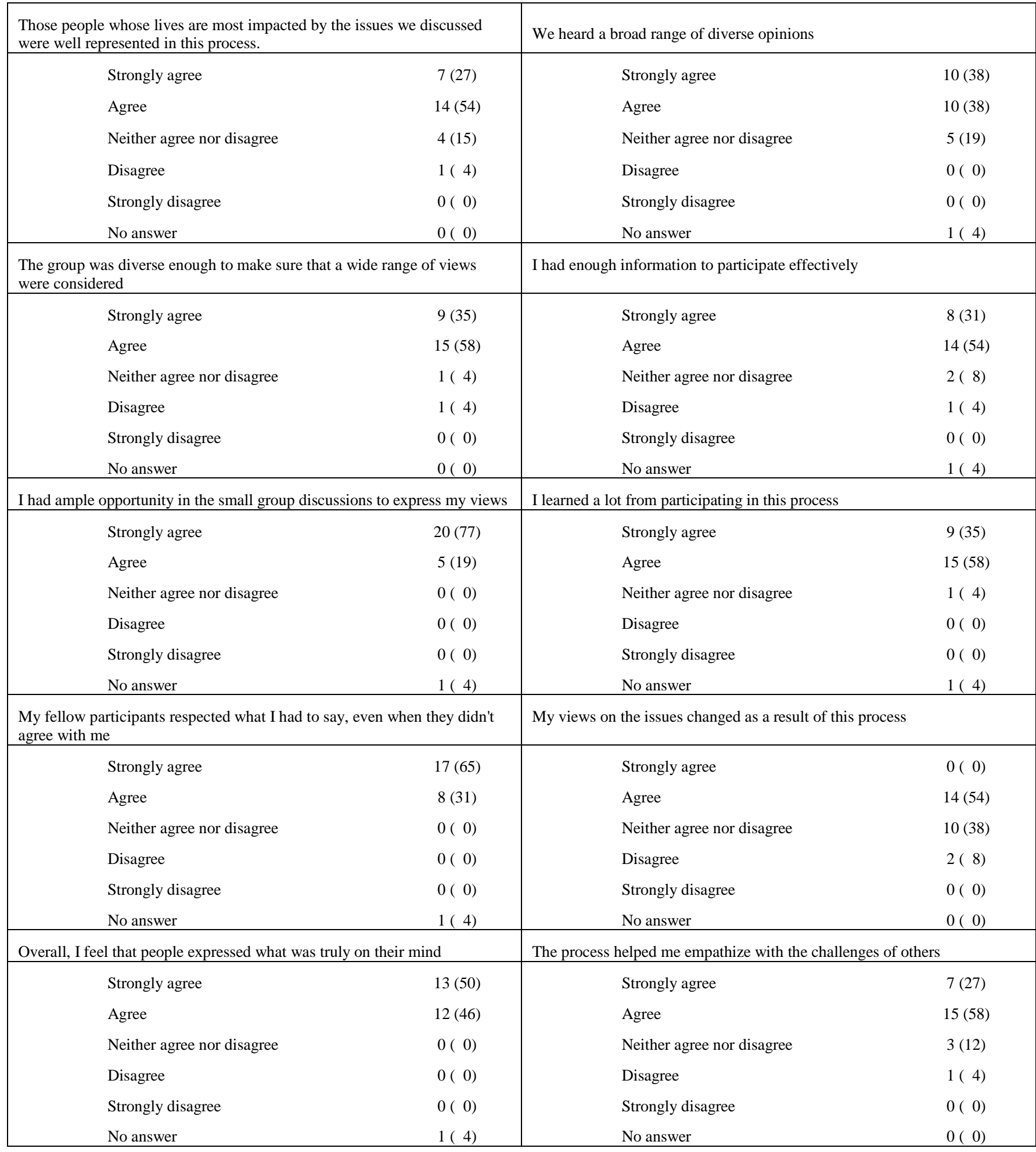

either satisfied (60\%) or very satisfied (20\%) and another $20 \%$ neutral. When clinicians were asked to evaluate how their perspective had been represented, $13 \%$ reported very satisfied; $60 \%$ satisfied and $20 \%$ neutral.

The process evaluation survey was completed by 26 participants (90\%). Mean (SD) age was 56 (11.0); 50\% were female and race was predominantly white (81\% white, 8\% African American, 12\% Asian). Of respondents, $58 \%$ self-identified as a health system leader, $4 \%$ as a health plan leader, $23 \%$ as a clinician, $12 \%$ as a patient and $4 \%$ as a community engagement expert. When asked about satisfaction with the overall process, $65 \%$ were satisfied 
and 35\% very satisfied. Respondents reported a wide range of familiarity with the issues that were the focus of the event: $54 \%$ very familiar, $42 \%$ somewhat familiar and $4 \%$ can't say. None reported being somewhat unfamiliar with the issues. Facilitators were generally felt to be unbiased (77\% completely unbiased; 19\% mostly unbiased; 4\% can't say).

Most survey respondents felt that the people most impacted by the issues discussed were well-represented by the deliberation process (27\% strongly agreed; 54\% agreed) and that the group was sufficiently diverse to ensure a wide range of viewpoints (35\% strongly agreed; 58\% agreed; Table 2). Respondents reported sufficient opportunity to express their views in the small group discussions. They felt their fellow participants respected what they had to say, even when having differing views. Participants agreed that people expressed what was truly on their mind and most noted having heard a broad range of opinions. Most, but not all, reported sufficient information to participate effectively. The majority agreed (35\% strongly agreed; 58\% agreed) that they learned a lot from participating. Over half noted that their views on the issues changed as a result of deliberation (54\%) and that the process helped them empathize with the challenges of others (27\% strongly agreed; $58 \%$ agreed).

\section{Discussion}

The HSLDP demonstrates that a deliberative forum can effectively engage health system/health plan leaders, along with other stakeholders, to prioritize CER questions of value to health systems. Participants reported high levels of satisfaction with the process. Key recommendations for stakeholder engagement were met, such as respect $(100 \%$ felt their fellow participants respected what they had to say), legitimacy (the stakeholder group was noted to be sufficiently diverse), fairness (decisions and rationales were made public), competence (participants had sufficient information to participate effectively) and accountability [20]. The event also demonstrated high deliberative quality [22]. Respondents noted that they had ample opportunity for active participation and adequate discussion, respect for others' opinions and awareness of different perspectives. Participants reported other markers of successful deliberation such as impact on knowledge (93\% learned from the experience) and attitudes (54\% noted their views on the issues changed) about the topics and that the process helped them to empathize with the challenges of others (85\% agreed) [22].

Our deliberative format design emphasized synchronous, face-to-face interaction between participants from different backgrounds. By bringing together stakeholders (health system leaders, clinicians and patients), the event created conditions for social knowledge to emerge. Social knowledge "depends on a personal relationship to other actors in the social world" and it "becomes the emergent property of a collectivity" when people communicate collaboratively to develop overlapping opinions on practical questions [29]. Social knowledge can be distinguished from the knowledge generated from survey data or public opinion polls, which aggregate static preferences expressed by individuals from within insular frames of reference. By contrast, when groups gather to deliberate, communication can trigger social learning processes that enable individuals to reassess and potentially revise their previously settled positions. When such deliberating groups draw upon the diverse horizons of experience and expertise shared by heterogeneous individuals, this collective learning process can yield social knowledge that is particularly robust.

A frequent outcome of group deliberation is opinion change [26,30], which was demonstrated at our event. Such findings suggest that the iterative discussion promoted learning and potentially fostered new insights as the various stakeholders learned of each other's perspectives.

A unique aspect of this event is its link to PaTH's and PCORnet's research agenda. PCORnet has subsequently proceeded with research on the high-priority topic area identified by PaTH's Health System Leaders' Demonstration Project - validating the outcome of this deliberation process. The genre of "empowered deliberation" is receiving heightened attention in the communication literature, with studies exploring how citizen and stakeholder deliberation can sometimes be linked, directly or indirectly, to decision-making within institutions [31]. Johnson and Gastil's scheme for ordering varieties of empowered deliberation highlights a gap in the literature regarding what they call "embedded and provisional" deliberation that takes place in a single, translocal event. Further study might usefully explore how the PaTH HSLDP can be viewed in this light, potentially illuminating the dynamic interplay between stakeholder deliberation and institutional decision-making.

Limitations include the fact that deliberative models require substantial time and effort to plan and execute [11] and CER teams typically do not include deliberation facilitators. Furthermore, stakeholders who do not routinely make health system decisions may find it difficult to reconcile personal advocacy agendas with a health system leader point of view. Varying the composition of the different end-users of the research among a stakeholder group could result in substantially different input into the CER process [11]. Yet, prior research indicates that while participants from historically under-represented demographic groups may place more value on or perceive greater impact from their participation in a deliberative forum than other participants, the proportion of discussants made up of a specific demographic group may minimally affect deliberative outcomes [22]. Furthermore, polarization (the systematic tendency of groups and the individuals who compose them to strengthen their pre-deliberation opinions) does not appear to be common using deliberative approaches [22]. The relatively small sample size that was assessed for opinion shifts limits our ability to examine subgroups. 


\section{Conclusion}

In summary, we found that a deliberative forum effectively engaged diverse health system/health plan leaders to prioritize CER topics. Productive conversation was fostered among stakeholders with various perspectives (e.g., health system leaders, patients, clinicians). The format was satisfactory to participants, who reported successful engagement and deliberative experiences. Opinions shifted during deliberation, suggesting that the in-person iterative discussion promoted learning. Deliberation may represent an important tool for incorporating diverse stakeholder into the CER process, such that participants can inform and learn from each other, while influencing a person-centered research trajectory.

\section{Acknowledgements and Conflicts of Interest}

The authors thank the participants of the Health System Leaders deliberation event for contributing their time and insights to this project. This work was funded through a Patient-Centered Outcomes Research Institute (PCORI) Award (CDRN-1306-04912) for development of the National Patient-Centered Clinical Research Network, known as PCORnet. The views presented in this work are solely the responsibility of the author(s) and do not necessarily represent the views of the Patient-Centered Outcomes Research Institute (PCORI), its Board of Governors or Methodology Committee. The authors declare no conflicts of interest.

\section{References}

[1] Slutsky, J., Sheridan, S. \& Selby, J. (2014). "Getting engaged". Journal of General Internal Medicine 29 (12) 1582-1583.

[2] Pignone, M. (2012). Challenges to implementing patient-centered research. Annals of Internal Medicine 157

(6) $450-451$.

[3] Sparud-Lundin, C., Josefsson, U., Berg, M., Hellstrom, A.L., Koinberg, I., Nolbris, M.J., Ranerup, A. \& Skärsäter, I. (2013). Use of participatory design in the development of person-centred web-based support for persons with long-term illness. European Journal for Person Centered Healthcare 1 (2) 369-380.

[4] Ammerman, A., Corbie-Smith, G., St George, D.M., Washington, C., Weathers, B. \& Jackson-Christian, B. (2003). Research expectations among African American church leaders in the PRAISE! project: a randomized trial guided by community-based participatory research. American Journal of Public Health 93 (10) 1720-1727.

[5] Jones, L. \& Wells, K. (2007). Strategies for academic and clinician engagement in community-participatory partnered research. Journal of the American Medical Association 297 (4) 407-410.
[6] Phillipson, J., Lowe, P., Proctor, A. \& Ruto, E. (2012). Stakeholder engagement and knowledge exchange in environmental research. Journal of Environmental Management 95 (1) 56-65.

[7] Longstaff, H. \& Secko, D.M. (2016). Assessing the quality of a deliberative democracy mini-public event about advanced biofuel production and development in Canada. Public Understanding of Science 25 (2) 252-261.

[8] Hall, T.E., Wilson, P. \& Newman, J. (2011). Evaluating the short- and long-term effects of a modified deliberative poll on Idahoans' attitudes and civic engagement related to energy options. Journal of Public Deliberation 7 (1) 1-30.

[9] Haywood, B.K. \& Besley, J.C. (2014). Education, outreach, and inclusive engagement: Towards integrated indicators of successful program outcomes in participatory science. Public Understanding of Science 23 (1) 92-106.

[10] Schmittdiel, J.A., Desai, J., Schroeder, E.B., Paolino, A.R., Nichols, G.A., Lawrence, J.M., O’Connor, P.J., Ohnsorg, K.A., Newton, K.M. \& Steiner, J.F. (2015). Methods for engaging stakeholders in comparative effectiveness research: a patient-centered approach to improving diabetes care. Healthcare 3 (2) 80-88.

[11] Workman, T., Maurer, M. \& Carman, K. (2013). Unresolved tensions in consumer engagement in CER: a US research perspective. Journal of Comparative Effectiveness Research 2 (2) 127-134.

[12] Concannon, E., McHugh, S., Healy, D.A., Kavanagh, E., Burke, P., Clarke Moloney, M. \& Walsh, S.R. (2014). Diagnostic accuracy of non-radiologist performed ultrasound for abdominal aortic aneurysm: systematic review and meta-analysis. International Journal of Clinical Practice 68 (9) 1122-1129.

[13] Selby, J.V. \& Slutsky, J.R. (2014). Practicing partnered research. Journal of General Internal Medicine 29 (Supplement 4) 814-816.

[14] Deverka, P.A., Lavallee, D.C., Desai, P.J., Esmail, L.C., Ramsey, S.D., Veenstra, D.L. \& Tunis, S.R. (2012). Stakeholder participation in comparative effectiveness research: defining a framework for effective engagement. Journal of Comparative Effectiveness Research 1 (2) 181194.

[15] Miles, A. (2017). From evidence-based to evidenceinformed, from patient-focussed to person-centered - The ongoing "energetics" of health and social care discourse as we approach the Third Era of Medicine. Journal of Evaluation in Clinical Practice 23 (1) 3-4.

[16] Reid, C. (2012). Developing a research framework to inform an evidence base for person-centered medicine: keeping the person at the centre. European Journal for Person Centered Healthcare 1 (2) 336-342.

[17] Miles, A. \& Asbridge, J.E. (2016). The chronic illness problem. The person-centered solution. European Journal for Person Centered Healthcare 4 (1) 1-5.

[18] Sibbald, S.L., Singer, P.A., Upshur, R. \& Martin, D.K. (2009). Priority setting: what constitutes success? A conceptual framework for successful priority setting. BMC Health Services Research 9, 43.

[19] Burgess, J., Stirling, A., Clark, J., Davies, G., Eames, M., Staley, K. \& Williamson, S. (2007). Deliberative mapping: a novel analytic-deliberative methodology to 
support contested science-policy decisions. Public Understanding of Science 16 (3) 299-322.

[20] Lavallee, D.C., Williams,. C.J., Tambor, E.S. \& Deverka, P.A. (2012). Stakeholder engagement in comparative effectiveness research: how will we measure success? Journal of Comparative Effectiveness Research 1 (5) 397-407.

[21] Hoffman, A., Montgomery, R., Aubry, W. \& Tunis, S.R. (2010). How best to engage patients, doctors, and other stakeholders in designing comparative effectiveness studies. Health Affairs 29 (10) 1834-1841.

[22] Carman, K.L., Maurer, M., Mallery, C., Wang, G., Garfinkel, S., Richmond, J. et al. (2013). Community Forum Deliberative Methods Demonstration: Evaluating Effectiveness and Eliciting Public Views on Use of Evidence. Executive Summary. (Prepared by the American Institutes for Research Under Contract No. 290-201000005.). Rockville, MD: Agency for Healthcare Research and Quality.

[23] Abelson, J., Forest, P.G., Eyles, J., Smith, P., Martin, E. \& Gauvin, F.P. (2003). Deliberations about deliberative methods: issues in the design and evaluation of public participation processes. Social Science \& Medicine 57 (2) 239-251.

[24] Califf, R.M. (2014). The Patient-Centered Outcomes Research Network: a national infrastructure for comparative effectiveness research. North Carolina Medical Journal 75 (3) 204-210.

[25] Karpowitz, C.F. \& Bansbridge, J. (2005). Disagreement and concensus: The need for dynamic updating in public deliberation. Journal of Public Deliberation 1(2) Article 2.

[26] Gutmann, A. \& Thompson, D.F. (2004). Why deliberative democracy? Princeton, NJ: Princeton University Press.

[27] Fisher, W.R. (1984). Narration as Human Communication Paradigm: The Case of Public Moral Argument. Communication Monographs 51, 1-22.

[28] Warren, M., Beauvais, E., Yaylaci, Ş., Mackenzie, M. \& Spada P. (2015). Participedia core participants' survey. Unpublished instrument. Copy in possession of authors.

[29] Farrell, T. (1976). Knowledge, consensus and rhetorical theory. Quarterly Journal of Speech 62, 1-14.

[30] Fishkin, J.S. (1997). The voice of the people: Public opinion and democracy. New Haven, CT: Yale University Press.

[31] Johnson, C. \& Gastil, J. (2015). Variations of institutional design for empowered deliberation. Journal of Public Deliberation 11 (1) Article 2. 